\title{
Air Embolism as a Complication of Venovenous Bypass During Liver Transplant for Diffuse Hemangiomatosis
}

\author{
J.S. Viana, E. Furtado, A. Romero, and A.L. Furtado
}

$\mathrm{V}$ ENOUS BYPASS has been extensively used during liver transplantation to decompress the surgical field and to reduce the hemodynamic effects of inferior vena caval clamping. Complications are rare $^{1}$ but some can be dangerous, ${ }^{2}$ such as the one observed in the reported situation.

\section{CLINICAL CASE}

A 48-year-old female patient, who was $162 \mathrm{~cm}$ tall and weighed 102 $\mathrm{kg}$, underwent a first liver transplant for diffuse hemangiomatosis. Preoperatively, she displayed a restrictive syndrome on respiratory function tests and increased dimensions of the right atrium and right ventricle on an echocardiogram. Renal function was normal and laboratory tests showed $10.3 \mathrm{~g} / \mathrm{L}$ hemoglobin, prothrombin time of 5.0 seconds above control, and normal values of platelet count, albumin, liver enzymes, and bilirubin.

On arrival at the operating room, the arterial pressure was $133 / 63 \mathrm{~mm} \mathrm{Hg}$ and heart rate 85 beats/min. Anesthetic induction was performed with thiopental, fentanyl, and succinylcholine; anesthesia maintenance utilized isoflurane vaporized in oxygen/air, fentanyl, and vecuronium. Monitoring included invasive arterial pressures, pulmonary artery catheter, pulse oximetry, and a Vigilance monitor (Edward-Baxter, Irvine, Calif, USA) for continuous evaluation of cardiac output and pulmonary artery oxygen saturation.

The native liver displayed remarkable dimensions and a strong tendency to laceration despite careful technique. For vascular decompression of the surgical field, a porto-femoro-axillary venous bypass was initiated with a vortex Biomedicus pump (Minn, USA), using transparent heparin-coated tubes and a disposable head pump (Jostra AG; Hirrlingen, Germany, reference BE-RF-32.)

An air embolism was detected a few minutes after the initiation of bypass by decreases in the end-tidal partial pressure of carbon dioxide $\left(\mathrm{PetCO}_{2}\right)$ from 26 to $16 \mathrm{~mm} \mathrm{Hg}$ and in the arterial saturation $\left(\mathrm{SaO}_{2}\right)$ from $97 \%$ to $93 \%$, with increased pulmonary artery pressures (PAP) from $34 / 19 / 26$ to $57 / 26 / 39 \mathrm{~mm} \mathrm{Hg}$, a slight decrease in systemic arterial pressure (AP) from $114 / 65$ to $102 / 75$ $\mathrm{mm} \mathrm{Hg}$, and an increased heart rate from 94 to 101 beats/min. Neither careful visual inspection nor the use of air detectors in the equipment were able to detect air inside the bypass system, but the femoral cannula showed a small hole that occurred due to a traumatic clamp used for cannula insertion. The hole was totally covered with an adhesive plastic band and the signs of air embolism disappeared within a few minutes.

While still on bypass, 2 hours and 50 minutes later, the AP decreased over 10 minutes from $112 / 65$ to $65 / 43 \mathrm{~mm} \mathrm{Hg}$ with increased PAP from 26/12/18 to $68 / 38 / 48 \mathrm{~mm} \mathrm{Hg}$ and central venous pressure (CVP) from 9 to $18 \mathrm{~mm} \mathrm{Hg}$. $\mathrm{PetCO}_{2}$ decreased from 27 to $12 \mathrm{~mm} \mathrm{Hg}, \mathrm{SaO}_{2}$ from $99 \%$ to $87 \%$, and heart rate from 112 to 87 beats/min (Fig 1). The adhesive band that covered the hole of the femoral cannula had become wet, lost its adherence, and allowed entry of air again, once more not detected by the equipment detectors or by visual inspection. Bypass was immediately stopped; the patient was treated with $50 \mu \mathrm{g}$ of epinephrine, $0.5 \mathrm{mg}$ of atropine, and $100 \%$ oxygen, recovering acceptable pressures in 12 minutes. Surgery continued and graft reperfusion was achieved 17 minutes after detection of this second episode of air embolism. No more hazardous events were observed; the patient was extubated some hours later. The further evolution was normal and no sequelae were observed after this occurrence. In our overall experience of bypass used in 125 (27\%) of 462 transplants, this is the sole case of air embolism with an origin in the bypass, leading to an incidence of $0.8 \%$ for this complication.

From the Departments of Transplantation and Anaesthesiology, Coimbra University Hospitals, Coimbra, Portugal.

Address reprint requests to Joaquim Viana, Department of Anaesthesiology, University Hospitals, Pct. Mota Pinto, 3000075 Coimbra, Portugal. E-mail: jvviana@huc.min-saude.pt
360 Park Avenue South, New York, NY 10010-1710 


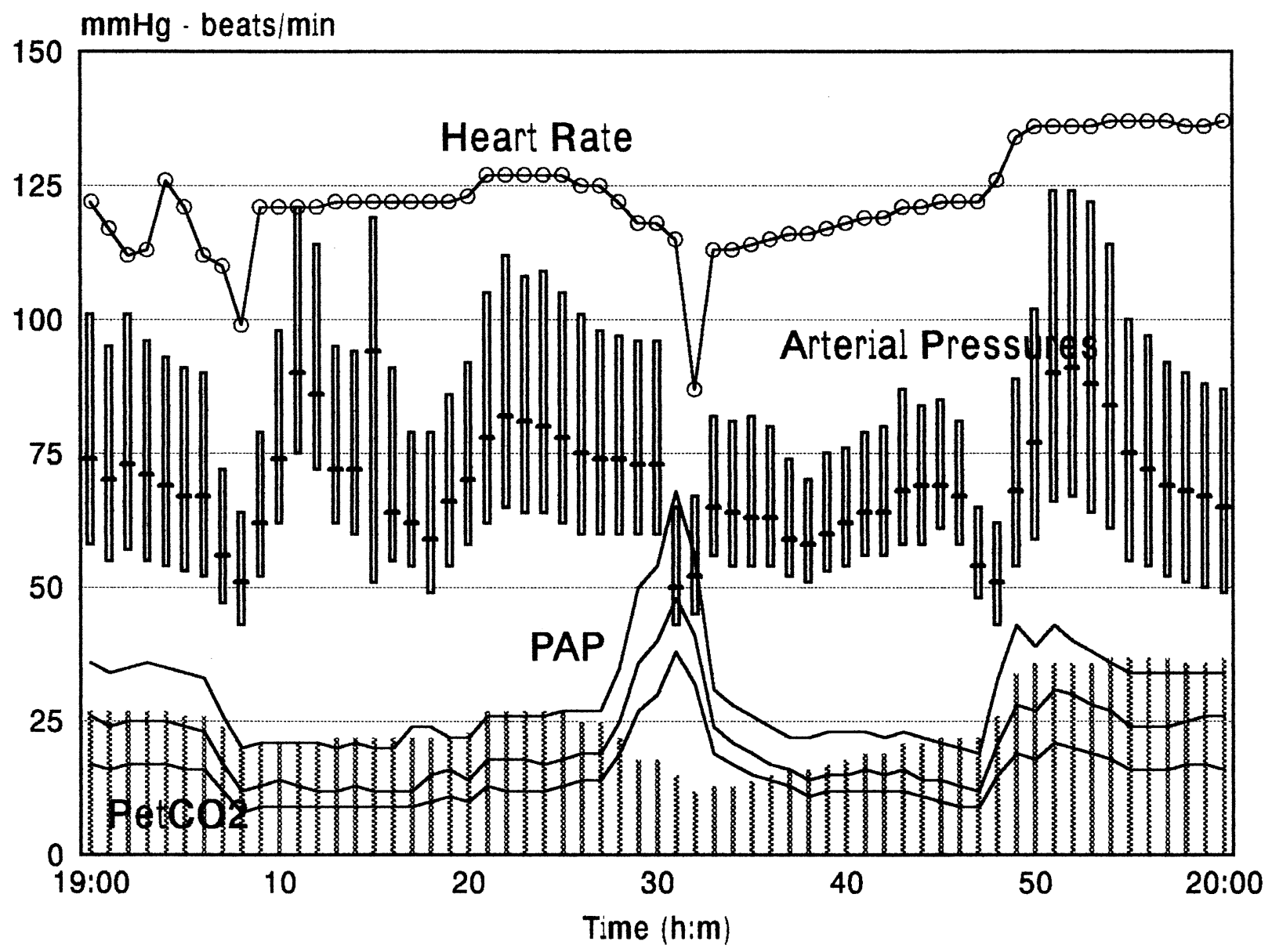

Fig 1. Records of the heart rate (straight line with dots), systolic, diastolic and mean arterial systemic pressures (high-low bars), systolic, diastolic and mean pulmonary pressures (straight lines in the bottom), and partial pressure of carbon dioxide in the expired gases (bars in the bottom).

\section{DISCUSSION}

Venovenous bypass has been extensively used in the past during liver transplantation. The advent of the piggyback technique, with preservation of inferior vena cava, made it a dispensable option in the great majority of cases. A certain degree of controversy now exists about its actual role in this surgical procedure.

The equipment traditionally used-a Biomedicus nonocclusive vortex pump-is, in general, safe: it imposes a speed to the blood that prevents coagulation in the system (and subsequent pulmonary thromboembolism) and the equipment contains air detectors that are effective to detect gas inside the tubes, stopping the pump and preventing abrupt air embolism. ${ }^{1}$

In spite of this equipment, our case shows that air detectors may fail to detect small entries of air-so insignificant that visual inspection is unable to see them-but great enough to accumulate in the lungs and produce right heart failure and circulatory collapse. This complication seems to be rare, ${ }^{2}$ but its potential danger demands that during bypass; independent of the detection of air in the system, attention must always be paid to early signs of gas embolism, namely a decreased expired carbon dioxide associated with an increased pulmonary artery pressures. The nonuse of nitrous oxide as an anesthetic is also recommended, ${ }^{2}$ as this gas has high solubility in comparison to the air oxygen or nitrogen and rapidly increases the volume of the gas bubbles in the pulmonary circulation. ${ }^{3}$

Furthermore, it is important to remark that some authors, ${ }^{4}$ in experimental conditions, have suggested that gas embolism may be observed inside the systems used for bypass during liver transplantation even without an external entry of air due to cavitation of the fluid, if particular conditions are met simultaneously: namely, a high pump speed and an obstruction before the pump producing a negative pressure. 
We doubt that the type of pump head played a role in this complication. We use one with a low priming volume when compared with traditional heads. It may be possible that higher volume pump heads cause micro-bubbles to coalesce consequently easing air detection. As far as we know, no studies exist about this subject, which in our opinion deserves further attention.

\section{REFERENCES}

1. Shaw BW, Martin DJ, Marquez JM, et al: Ann Surg 200:524, 1984

2. Khoury GF, Mann ME, Porot MJ, et al: Anesthesiology 67:848, 1987

3. Munson ES: Anesth Analg 50:785, 1971

4. Arcari M, Phillips SD, Gibbs P, et al: Transplantation 68:150, 1999 